\title{
OCUPACIÓN HUMANA Y LA PRÁCTICA DE LOS TERAPEUTAS OCUPACIONALES EN LA AMAZÔNIA EN PARÁ: UNA PERSPECTIVA FENOMENOLÓGICA
}

\author{
HUMAN OCCUPATION AND OCCUPATIONAL THERAPIST \\ PRACTICE'S IN PARÁ AMAZON: A PHENOMENOLOGICAL \\ PERSPECTIVE
}

\section{Resumen}

El trabajo tiene el propósito de entender el significado que terapeutas ocupacionales asignan a las ocupaciones humanas y las relaciones que establecen con sus prácticas profesionales. Esta es una orientación cualitativa fenomenológica que une conocimientos teóricos y prácticos de la Ciencia de Ocupación y de la Terapia Ocupacional. Los participantes de este estudio fueron diecisiete terapeutas ocupacionales que trabajan con el cuidado y educación relacionados a la función pública en el estado de Pará, Brasil. Los resultados indican que cada terapeuta ocupacional entrevistado diseña y ejerce su propia Terapia Ocupacional, de acuerdo a su área de trabajo, la población atendida, objetivos de intervención, bases teóricas en las cuales se sustenta y sus creencias y valores personales. Los profesionales entrevistados comparten la idea de que las ocupaciones son constituyentes de la vida humana, entendiéndose que esta tiene la capacidad de influir en la salud, calidad de vida y participación social de las personas; la valoración de las intervenciones estructuradas en lo que es significativa, relevante y necesario para sus pacientes. Esta investigación ha permitido la elaboración de preguntas y reflexiones sobre los fundamentos teóricos y las acciones prácticas emprendidas en el ámbito de la Terapia Ocupacional; esto, en el supuesto de que tanto los estudios de campo epistemológico y en clínicas son necesarias para el desarrollo de diálogos, porque compartir es la comprensión que la Terapia Ocupacional Brasileña, y en particular el desarrollo de la Terapia Ocupacional en la región amazónica es única, tiene mucho que aportar al desarrollo de la profesión en los ámbitos regional, nacional e internacional.

\section{Palabras Clave}

Ocupación. Terapia Ocupacional. Fenomenológica. Ciencia de La Ocupación. 


\begin{abstract}
This paper aims to understand the meaning that occupational therapists attribute to occupations and the relationships they build with their professional practices. This is a qualitative phenomenological orientation that combines theoretical and practical knowledge of the science of occupation and occupational therapy. The participants were seventeen occupational therapists who work in care and education of occupational therapy linked to public service in the state of Pará, Brazil. It was observed that each occupational therapist is researched and is planning its own occupational therapy, according to its area of operation, population served, their own beliefs and values, and perspectives and theoretical predictions. The subjects share the idea that occupations are constituents of human life, understanding them to influence the health, quality of life and social participation of individuals, valuing structured interventions on what is meaningful, relevant and necessary for their patients. This research enabled the development of questions and reflections about the theoretical foundations and practical actions carried out in the field of occupational therapy. It is believed that studies both in the epistemological field and in the clinical are required for the development of these dialogues, because sharing is the understanding that the Brazilian occupational therapy, and particularly the development of occupational therapy in the Amazon region is unique, and very contribute to the development of the profession on the regional, national and international.
\end{abstract}

\title{
Key-Words
}

Occupation. Occupational Therapy. Phenomenology. Science of Occupation.

\section{INTRODUCCIÓN}

El desarrollo de la Terapia Ocupacional como profesión siempre sufrió influencia directa de distintas perspectivas que envuelven las comprensiones de la ocupación y sus desdoblamientos en los variados contextos. De este modo, fueron muchos los conocimientos venidos de otros campos, por ejemplo las ciencias sociales, ciencias biológicas, ciencias de la salud y la propia (1). Sin duda, este "préstamo" influenció en la versatilidad y variabilidad de los campos de amplitud de la Terapia Ocupacional, repercutiendo en muchos aspectos.

Uno de esos aspectos sorprendente está relacionado al acercamiento con los modelos médico y psicológico, así como con las actuales modalidades de la ciencia, favoreciendo un alejamiento de la filosofía de la profesión con relación a las ocupaciones, y dificultando el desarrollo de un cuerpo de conocimiento propio, en determinado momento socio histórico. Sin embar- go, creemos que la apropiación de conocimiento de áreas distintas haya favorecido el desarrollo de un mirar multidimensional y complejo sobre las ocupaciones, permitiendo el florecimiento de una ciencia propia enfocada en este objeto.

En ese contexto, la Ciencia de Ocupación, se presenta como una disciplina emergente que comparte valores con la Terapia Ocupacional y comprende el ser humano como un ser ocupacional, uniendo conocimientos de diversas áreas, sea de las ciencias sociales y humanas, sea de las biológicas, de la salud, de las naturales y tecnológicas, entre otras. Direccionar la relación entre el compromiso en ocupaciones y la vida humana, particularmente, como esta relación influencia la salud, bienestar y la participación social (2) (3).

En Brasil, sin embargo, existe un fuerte rechazo a las comprensiones de ocupaciones producidas en el escenario internacional, desarrolladas principalmente en países anglosajones, debido a los paradigmas pre- 
dominantes y principios que orientan la practica de los terapeutas ocupacionales y sus elaboraciones teóricas en estos países (4) (5) (6). Se nota, no obstante, que esos rechazos son dirigidos, principalmente a modelos que presentan conceptos, abordajes y perspectivas centradas en las ocupaciones, que no son aplicables con la realidad brasileña.

En el escenario de la Terapia Ocupacional Brasileña que se fundamenta en estudios centrados en la ocupación, las producciones son escasas. En la Amazonía, específicamente en el Estado de Pará, mucho menos aún se produce cerca de la Ciencia de la Ocupación, sus presupuestos y sus contribuciones al desarrollo y fundamento de la Terapia Ocupacional. Una iniciativa positiva para el cambio de este escenario ocurrió hace alrededor de dos años, cuando un grupo de terapeutas ocupacionales vinculados con la Universidad del Estado de Pará (UEPA), estuvieron participando en la Escuela de Terapia Ocupacional de la Universidad de Chile, en Santiago de Chile, de un curso de Ciencia de la Ocupación y fundaron en Belém de Pará, el Centro de Estudios de Ciencia de la Ocupación (LECO), que entre sus varias iniciativas se aboca al estudio y divulgación del fundamento teórico que sostiene a esta ciencia. En este contexto se presenta la investigación de que trataremos en este artículo.

La contribución de este estudio para la construcción de conocimiento de la profesión, se basa en la intención de investigar aspectos que introducen el objeto profesional de la terapia ocupacional, la ocupación, bajo un punto de vista singular: el significado atribuido por aquellos que ejercen la profesión, los terapeutas ocupacionales.

En este artículo, por lo pronto, presentaremos, los resultados de una investigación, realizada junto a los terapeutas ocupacionales de la Amazonía Paraense, con el objetivo_de comprender el significado que estos profesionales atribuyen a las ocupaciones y las relaciones que establecen con sus prácticas clínicas.

Investigar esa temática se vuelve oportuno en la medida que evidencia cuestiones inherentes a la estructuración y consolidación de la profesión en el escenario local, pues refleja la percepción que los profesionales tienen en relación a los constructos teóricos de su práctica clínica, ampliando las posibilidades de diálogo en la frontera de la interdisciplinaridad.

\section{Metodo}

El transcurso metodológico fue constituido de tres etapas. La primera consistió en el análisis bibliográfico acerca de las principales cuestiones teóricas que envuelven el concepto de ocupación bajo la perspectiva de la Ciencia de Ocupación y su articulación con profesión de Terapia Ocupacional. Ese análisis se desarrollo utilizando libros, artículos científicos, periódicos específicos, sitios de Internet, tesis y disertaciones.

La segunda etapa fue constituida por investigación de campo, por medio de cuestionario semidirigido, con terapeutas ocupacionales que trabajan en instituciones públicas de asistencia y enseñanza en el municipio de Belém - Pará.

En la tercera etapa, los datos fueron analizados, articulando los presupuestos teóricos del campo de conocimiento y la perspectiva de los investigadores.

Se trata de una investigación cualitativa de orientación fenomenológica que se articula en la frontera del campo interdisciplinario; entre el saber de los sujetos investigados, el campo de conocimiento de la Ciencia de Ocupación, la Terapia Ocupacional y la perspectiva de los investigadores acerca de ello.

La opción por el método cualitativo se hizo a partir del entendimiento de que esta perspectiva se emplea en la concepción traída de las ciencias humanas, según las cuales "no se busca estudiar el fenómeno en su totalidad, sino entender su significado individual o colectivo para la vida de las personas" (7). En ese sentido, el significado atribuido a tiene función estructurante, o sea, alrededor de que las cosas significan, las personas organizan de cierto modo sus vidas, sus comportamientos y acciones. Lo que las "cosas" (fenómenos, manifestaciones, ocurrencias, hechos, eventos, experiencias, ideas, 
sentimientos, temas) representan, perfectamente la vida de las personas.

Junto a esta orientación cualitativa, la posición fenomenológica de los investigadores orienta a la conducción metodológica de este estudio en búsqueda de la descripción de la estructura y del significado implícito de la experiencia humana partiendo de la comprensión del vivir, siendo esta comprensión vuelta para los significados expresos por los sujetos de aquello que está siendo investigado (8). El abordaje fenomenológico busca aprender aspectos del fenómeno por medio de lo que dicen los sujetos, pues, "cuando los otros describen aspectos del fenómeno, ellos los describen como los notan, en el deseo de comunicar sus percepciones" (9).

La muestra de esta investigación se constituye de 20 (veinte) terapeutas ocupacionales, vinculados al equipaje público de asistencia a la salud y enseñanza del Gobierno del Estado de Pará. La selección de esas instituciones se hizo por el hecho de que tienen, un número considerable de estos profesionales en sus funciones. La distribución de los informantes en esos locales fue equitativa, o sea, 10 (diez) participantes originarios de los equipamientos de salud y los otros 10 , del equipamiento de enseñanza.

Las normas de inclusión en el estudio fueron:) tener 2 años o más de formación profesional; b) aceptar y firmar el consentimiento libre e informado (TECLE); y c) ser terapeuta ocupacional perteneciente a los equipamientos de salud pública del Gobierno del Estado de Pará o al equipamiento de enseñanza superior de este gobierno. Esta investigación fue aprobada por la Comisión de Ética y Investigación con seres humanos de la Fundación Hospital de Clínicas Gaspar Vianna, donde fue hallada de acuerdo a las normas internacionales específicas.

Como instrumento de recolección de datos se diseño un cuestionario semiestructurado. Las preguntas fueron dirigidas para demandas de orden subjetiva del profesional en el pleno ejercicio de su función. Destacamos aún que la investigación no pretendió averiguar aspectos que envolvían la dinámica laboral u obtener cualquier dato de orden institucional.

En lo que se refiere al tratamiento de los datos, se utilizó el proceso de categorización. La palabra categoría se refiere a un "concepto que abarca elementos o aspectos con características comunes o que se relacionan entre sí" (10). En los estudios fenomenológicos se utilizan esta forma de presentación y discusión de los datos, como método de análisis. Las categorías son empleadas para establecer clasificaciones, reuniendo elementos, ideas o expresiones que se desarrolla alrededor de los sentidos que los sujetos atribuyen al fenómeno estudiado, como veremos a continuación.

\section{Resultados}

De los cuestionarios distribuidos, 17 (diecisiete) fueron devueltos. Para analizar los datos obtenidos, creamos categorías de análisis, y forma de trabajar los datos, para ello, se consultaron diversos autores (11) (12) (13). Cada categoría fue estructurada a partir de la articulación entre los datos empíricos obtenidos, la perspectiva fenomenológica que orientó la investigación y la inmensa producción teórica del campo de conocimiento de la Ciencia de Ocupación y de la Terapia Ocupacional. Los fragmentos transcriptos de los cuestionarios fueron identificados por TO1, TO2, etc. Haciendo referencia al cuestionario del primero y del segundo terapeuta ocupacional, en orden a devolver el instrumento completado. Las transcripciones fueron hechas integralmente, manteniéndose a la construcción ortográfica, gramatical y semántica, así como los destaques en el texto de opción de los propios informantes.

Los datos posibilitaron el desarrollo de tres categorías de análisis: a) definiciones y el saber: una identidad profesional en construcción; b) la ocupación desde el punto de vista de terapeutas ocupacionales; y c) ocupación y practica del terapeuta ocupacional. 


\section{a) Definición y saber: una identidad profesional en construcción}

En el campo de las proposiciones acerca de una definición de profesión, notamos que la mayoría de los informantes desarrolla una perspectiva conceptual que, generalmente, se presenta en armonía con su área de experiencia práctica e sus fundamentos teóricos.

Profesión del área de salud que busca desarrollar o estimular las potencialidades humanas (TO1 Salud Mental)

Profesión del área da salud con actuación en el campo social y educación que mira promocionar las habilidades funcionales del individuo para el desempeño ocupacional en los diversos contextos (casa, escuela, trabajo etc.) (TO9- Rehabilitación Física)

Profesión del área de salud que atiende al ser humano dentro de sus necesidades biopsicosociales buscando una mejor calidad de vida (TO14 - Hospital)

Esas percepciones distintas de Terapia Ocupacional, tal vez estén relacionadas con la tesis de que cada profesional desarrolla su propia definición con respecto a la profesión y esta, por su vez, trae en su objetivo, líneas de experiencias practicas, del área de actuación, de los fundamentos teórico metodológicos, de las creencias y perspectivas con relación al hombre, la salud y al proceso terapéutico (5).

Terapia Ocupacional es una profesión del área de salud que busca promocionar la calidad de vida por medio de la habilitación/ rehabilitación de las personas con el objetivo de favorecer la realización de sus actividades cotidianas de forma más independiente y satisfactoria... (TO16)

Profesión que, a través de la actividad humana, busca la mayor autonomía del sujeto en sus ocupaciones en diferentes contextos (TO3)
Posee en el "hacer", en la ocupación humana su instrumento de actuación. Parte del principio que este "hacer" es transformador (TO7)

Trabaja en la asistencia del "hombre ocupacional" donde tanto en la promoción, prevención y recuperación de la salud utilizan como recurso científico las actividades humanas (TO13)

En la formulación de los conceptos presentados se valoran, compresiones divergentes, de la ocupación, actividad y/ o hacer humano en la actuación del terapeuta ocupacional. Creemos que la manera cómo cada profesional comprende su profesión, repercute directamente en sus practicas cotidianas y influye en la perspectiva que pacientes, equipo y sociedad desarrollan acerca de la profesión y de su papel social.

Esa multiplicidad de posicionamientos alrededor de la definición de la profesión no es reciente y tal vez esté lejos de un consenso. En la literatura especializada encontramos varios teóricos que discuten esa cuestión bajo en distintas opiniones. Como ejemplo, podemos citar Medeiros que defiende la idea de que una profesión debe ser definida y diferenciada por su objetivo (14), en contrapunto a las tesis de Benetton y Soares que se posicionan bajo el argumento de que la diferencia de la profesión ocurre a través de la aplicación de los instrumentos y metodología de trabajo (15) (16). Caníglia, por su vez, defiende ese diferencial basándose en discusiones acerca del objeto profesional (17).

Las percepciones de los informantes sobre la profesión, su lugar académico y clínico, manifiestan una multiplicidad de puntos de vistas sobre la práctica de Terapia Ocupacional, bajo fuerte influencia teórica de sus respectivos campos de actuación. Los contenidos expresados apuntan a lo que muchos autores ya vienen reflexionando a lo largo de los años: que estamos hablando y haciendo no una, más varias terapias ocupacionales.

Lejos de querernos apuntar una "mejor" o "peor" perspectiva sobre cómo hacer profesionalmente, creemos que tal vez, todas las aplicaciones tengan algo a sumar en el conjunto de características que nos identi- 
fican. Así, creemos en una Terapia Ocupacional como profesión actuante tanto en la esfera de salud, como en la educación o en el campo social, que articula una gama de conocimientos originarios de varios campos, y comprende el hombre como un ser ocupacional capaz de influenciar su salud, participación social y calidad de vida a través de sus ocupaciones.

\section{b) La ocupación bajo la óptica de terapeutas ocupacionales}

Desde sus orígenes de Terapia Ocupacional, el termino ocupación ha sido usado para referirse a la participación de las personas en actividades cotidianas, tales como auto cuidado, trabajo, ocio, entre otros. Sin embargo, comprendemos que dentro de la literatura de Terapia Ocupacional, su significado ha sido ambiguo debido el uso similar con otros conceptos (18).

Así, notamos la predominancia de la utilización del término actividad o actividades para describir ocupación. Entendemos que esta situación ha tenido gran repercusión en la literatura mundial y nacional de la disciplina, y aún, en la comprensión que las profesiones han desarrollado acerca de esta construcción y de su uso en el campo de Terapia Ocupacional. En ese contexto, los participantes de la investigación, cuando se refieren a las ocupaciones, abarcan, de una forma general, las diversas acciones desarrolladas por los individuos dentro de un determinado contexto.

Todo tipo de actividad en la cual el ser humano se ocupa (TO7)

Todas las actividades realizadas por el hombre en su contexto social (TO11)

Es el conjunto de acciones que movilizan el hombre, que poseen significado y/ o significación en la vida de estas personas y que son capaces generar transformación (TO16)

En ese sentido, Lentin destaca que el termino actividad ha asumido significados relacionados a los campos de interés y acción de Terapia Ocupacional (19). Para Hinojosa y Kramer, los conceptos de ocupación, actividad con propósito y función se han usado indiferentemente en la literatura y practica de Terapia Ocupacional, y, en virtud de que están relacionados desde su inicio con la profesión, presenta igualdades en sus significados (20).

En el ámbito brasileño, Toyoda y Akashi identificaron que el termino actividad es entendido como acción, y, esta, es entendida como hacer. La ocupación, por a su vez, es también entendida conforme acción, pero también expresión humana (21).

Destacamos también que algunos sujetos observan la importancia del contexto y/ o contextos en el desarrollo de las ocupaciones, como nos presenta la "Estructura y Practica de la Terapia Ocupacional" (22).

Toda y cualquier actividad realizada por una persona, considerando sus contextos de trabajo, ocio y actividades sociales... (TO4)

Actividades desempeñadas por el individuo en su cotidiano en los diversos contextos en que se presenta (TO9)

Todas las actividades realizadas por el hombre en su contexto social (TO11)

Algunos estudios han presentado importantes conclusiones sobre la relación entre ocupaciones y contextos, destacando la cuestión cultural (23) (24). Estas investigaciones afirman que la perspectiva de Terapia Ocupacional desarrollada en el occidente es muy diferente de la que fue desarrollada en el oriente, debido, principalmente a la cultura, que influencia la comprensión de la ocupación y ello se refleja sobre la práctica de los profesionales y las investigaciones en el campo.

Una afirmación importante identificada en las respuestas de los sujetos respecto a la comprensión de ocupación, se ve inmersa en el cotidiano de las personas, donde las ocupaciones desarrolladas dan sentido, estructuran y permiten la identificación del sujeto en ese contexto.

Todo lo que penetra en la vida de los individuos desde la niñez hasta la madurez. El jugar, las relaciones de trabajo, el ocio (TO10) 
Toda y cualquier actividad que hace parte del cotidiano de cada individuo y que da sentido a ese cotidiano, variando de acuerdo con la edad, sexo y raza (TO8)

Hacen parte de nuestras necesidades, se refieren al hacer cotidiano o no cotidiano y que varían de persona para persona de acuerdo con su contexto individual y sociocultural (TO13)

En la perspectiva brasileña, esta relación ha sido presentada, entre otros, por Castro, Lima y Brunello, que, entienden lo cotidiano como la vía para orientarnos, siendo aquello que es dado cada día, donde las actividades y cuestiones rutinarias componen los acontecimientos diarios de la vida de los individuos (25). En el panorama internacional, Hasselkus ha evidenciado esa preocupación, afirmando que las ocupaciones cotidianas son los modos fundamentales por los cuales las personas organizan el mundo al cual ellas viven, constituyendo el ritmo de su vida diaria (26).

Demuestran que las ocupaciones asumen peculiaridades individuales, destacando la naturaleza ocupacional del ser humano, capaz de caracterizar aspectos relacionados a la existencia de los individuos, los sujetos de la investigación nos permiten especular una comprensión de ocupación como elemento constituyente de la vida de los sujetos.

El termino se refiere a toda y cualquier actividad realizada por una persona, considerando sus contextos de trabajo, ocio y actividades sociales, o sea su esencia humana (TO4)

Las ocupaciones humanas son esenciales para la vida del individuo. Es a través de ellas que las personas construyen sus relaciones, sus papeles en la sociedad. Las ocupaciones son dinámicas y se desarrollan a lo largo de toda la vida (TO9)

Las ocupaciones humanas son imprescindibles para nuestro bienestar y salud. Ante a ellas nosotros nos constituimos como personas, formamos nuestra identidad, nos relacionamos y nos realizamos, satisfaciendo nuestras necesidades humanas (TO13)
Los discursos presentados nos direccionan a comprender una perspectiva de ocupación que transciende el cotidiano y logra un valor terapéutico como favorecedor a la transformación de la vida, capaz de influenciar la salud, calidad de vida y participación de los sujetos en los contextos; como argumenta William Rush Dunton, uno de los fundadores de la Terapia Ocupacional, que la ocupación es tan necesaria para la vida cuanto la comida y la bebida (27).

Otra relación que se destaca en el discurso de los informantes es aquella que se establece entre ocupación y salud, donde algunos sujetos presentan una visión que relaciona el estado de salud y el desarrollo de ocupaciones, defendiendo la tesis de que una alteración en la salud afecta al desempeño de ocupaciones y viceversa.

Entiendo que existe una estrecha e intima relación entre ellas, pues cuando la salud está debilitada por alguna razón, el individuo entra en sufrimiento por no poder desempeñar las ocupaciones que lo identifican como sujeto (TO3)

Considerando que los diversos aspectos de las Ocupaciones Humanas (social, cultural, económico, biológico, filosófico...) son determinantes para la salud, la ocupación irá influenciar directamente en la salud (Q.5)

Existe siempre una estrecha relación entre el mantenimiento de un buen estado de salud y el equilibrio de las ocupaciones realizadas. Así también existe relación entre alteraciones de la salud y falta de equilibrio (exceso o falta de ocupaciones, falta de identificación con la actividad, frustración, etc.) (Q.8)

El panorama presentado refuerza la relación entre ocupaciones, salud, calidad de vida y participación, como uno de los principales focos de estudios en Terapia Ocupacional y Ciencia de Ocupación en los últimos años, debido, principalmente, al desarrollo y expansión de estos campos. 
Como ejemplos, podemos citar el estudio de Law, Steinwender y Leclair que revisaron en la literatura investigaciones sobre la relación entre ocupación, salud y bienestar, identificando pocos estudios sobre esta relación (28); el trabajo de Clark y colaboradores sobre los efectos del estilo de vida de personas con lesiones en la medula espinal y el desarrollo de úlceras de presión (29); Y los estudios de Jackson y colaboradores y Rudman, Cook y Polatajko sobre el potencial de las ocupaciones como favorecedoras a la salud y a la participación de los sujetos (30) (31).

Finalmente, destacamos la necesidad presentada por algunos sujetos de la investigación, de estudios más profundos, con respecto a este constructo tan fundamental para la Terapia Ocupacional que es la ocupación.

Comprensión del objeto de estudio es buena, pero, aún confusos necesitamos ser más objetivos y claros en cuanto a nuestro objeto de estudio y actuación. Así, nuestras intervenciones serán más efectivas (TO7)

Objeto de estudio es amplio y merece constante estudio y perfeccionamiento por parte de los profesionales (TO10)

Objeto de la Terapia Ocupacional precisa ser mejor definido con miras a congregar las diversas actuaciones y practicas de esta ciencia (TO9)

Yerxa hace casi 50 años, apuntó a este hecho, incitando el desarrollo de una Terapia Ocupacional "autentica", construida sobre conocimientos genuinos, orientados por principios, creencias, valores, sistematizados a través de investigaciones y estrictamente relacionados con la practica (32). En el ámbito nacional, Medeiros y Caníglia apuntan la necesidad de construcción de una epistemología propia de la Terapia Ocupacional, principalmente, en el campo brasileño, que presenta peculiaridades diferentes de otros países (6) (17).

\section{c) Ocupación y la práctica del terapeuta ocupacional}

Los terapeutas ocupacionales investigados demostraron que cuando planifican sus intervenciones, consideran aspectos relacionados a la cotidianeidad de los sujetos, dando prioridad a sus necesidades y deseos.

Al planificar la intervención procuro considerar los aspectos físicos, mentales y sociales de los sujetos (TO1)

Aspectos culturales, sociales, económicos, actividades que realizaba antes de la patología, expectativa para el futuro (TO5)

Grado de autonomía e independencia, contexto socio histórico y educacional, interés cultural, medio, hábitos, necesidad (TO6)

Esta constatación nos permite exponer que los profesionales investigados estructuran sus intervenciones pautadas en una multiplicidad de aspectos de las ocupaciones; éstas pueden relacionarse tanto con factores de tipo personal, individual y subjetiva, y al mismo tiempo con factores de orden externa como contextos, valores da sociedad, demandas de las ocupaciones, entre otros.

Con relación a la utilización de fundamentos teóricos como medio de la practica, sean modelos, abordajes, estructuras de referencia, entre otras, notemos que cada profesional presenta una forma peculiar de apropiarse de determinados referenciales y aplicarlos a la practica cotidiana, sin embargo, la mayoría cree que no debemos restringirnos a una única perspectiva y así conglomerar varias posibilidades de acuerdo con cada caso.

No uso un modelo. Mezclo modelos distintos y sus contenidos, para tener mayor flexibilidad y transitar entre los mismos de manera para que se aproveche y asimile los mejores contenidos, no me restrinjo apenas a un número reducido de modelos (TO4). No utilizo ningún modelo para la practica (TO7). 
Elijo los modelos de acuerdo con la necesidad, momento de la terapia, área de actuación buscando orientaciones teóricas y a veces practicas que subsidien mi actuación (TO8).

Los modelos son utilizados de acuerdo con la demanda de cada cliente (TO9).

En el campo teórico, principalmente en al ámbito nacional, encontramos un fuerte reproche para la utilización de modelos teóricos. Mângia y Medeiros reprochan la implementación de esas perspectivas, pues consideran que son perspectivas de hombre, salud y ocupación diferente, que no se ajustan con la realidad brasileña, pero refuerzan la importancia de construir nuestras acciones amparadas teóricamente (5) (6).

Sobre la percepción que cada profesional desarrolla acerca de sus contribuciones en la vida de su público, destacamos que la mayoría comprende tales contribuciones a partir de informaciones que parten de sus clientes, que identifican mejorías en el desarrollo de ocupaciones en su vida cotidiana, en la ayuda para restablecer la salud y de su participación exitosa en los contextos.

Creo que $80 \%$ de los pacientes que se someten al tratamiento logran beneficiarse con la terapia y logran llevar los beneficios de las sesiones para su cotidiano, sea en el punto de vista de una "recuperación física", sea en las relaciones interpersonales, en la autoestima e autoconciencia... (TO8)

Los pacientes relatan que se sienten mejor en su cuadro físico funcional, pues están haciendo actividades que antes de realizar el tratamiento no conseguían desempeñar... (TO9)

Mi contribución es positiva, pero discreta ante los obstáculos cotidianos en el universo de la vida de mi paciente que necesita alcanzar innumeras batallas como ser humano actuante e ciudadano. Pero me quedo feliz por posibilitar nuevas perspectivas, nuevos logros, hacer emerger las posibilidades y facilitar las transformaciones... (TO16)
En este panorama, comprendemos que Terapia Ocupacional, actualmente, viene sufriendo presiones tanto internas como externas, con respecto a la presentación de resultados efectivos de su proceso de intervención. Externamente, políticas públicas, planes de salud, así como otras categorías profesionales vienen solicitando resultados más efectivos y concretos de las intervenciones terapéuticas ocupacionales. Internamente, los propios profesionales se han cuestionado respecto a sus resultados. En este sentido, observamos el desarrollo de propuestas de modelos de evaluación, intervención y colección de resultados en el área, con el intento de demostrar la eficiencia de las intervenciones.

Sin duda, exponemos que esta verificación no puede ocurrir solamente por protocolos normalizados, muchas veces monopolizados, que desconsideran los aspectos individuales y subjetivos, así como los contextos histórico, social y cultural de los sujetos.

\section{DISCUSIÓN}

La poca bibliografía en el ámbito nacional, teniendo en cuenta la ocupación como elemento fundamental de Terapia Ocupacional, nos llevó a recurrir a la producción de autores extranjeros para subsidiar esta investigación, lo que posibilitó la articulación entre los significados atribuidos por los sujetos y las perspectivas de los investigadores.

La riqueza de los datos de la investigación nos permite deducir que muchas otras cuestiones aún podrían ser explotadas a partir del material empírico. Sin embargo, las unidades mencionadas manifiestan los principales contextualismos que consideramos pertinentes discutir y que atendieron al propósito de este trabajo.

De los resultados presentados, algunos aspectos merecen una mayor reflexión en el sentido de sus repercusiones en la construcción de conocimiento de Terapia Ocupacional en el contexto regional.

Una primera cuestión está unida a la percepción de que cada terapeuta ocupacional investigado hace y concibe su propia Terapia Ocupacional, de acuerdo 
con su área de actuación, población atendida y fundamentos teóricos influenciados, de una manera singular, por principios y valores fundamentales de la profesión. Comparten la idea de que las ocupaciones son elementos constituyentes de la vida humana, comprendiéndolas como capaces de influenciar la salud, la calidad de vida y la participación social de los individuos.

Independiente de los campos de actuación y de las perspectivas teóricas adoptadas, estos profesionales le dan un potencial terapéutico a las ocupaciones, valorando intervenciones estructuradas en lo que es significativo, relevante y necesario para sus pacientes. Entienden que la profesión presenta una importante contribución para la vida de los mismos, sin embargo, su reconocimiento, tanto por el equipo como por los clientes, aún está en proceso de madurez.

La investigación envolvió sujetos vinculados con la enseñanza y la intervención clínica en Terapia Ocupacional. Sin embargo, exponemos que esa categorización efectivamente sirvió apenas como criterio de inclusión, pues la mayoría de los docentes, de alguna manera están también vinculados con asistencia sea en un área independiente o en alguna de las instituciones públicas de las cuales son oriundos los informantes de la investigación. Así, tal vez, los discursos presentados por los informantes, sobre algunas cuestiones, presenten ideas que se armonizan en el campo teórico y práctico de la profesión.

Aunque valoremos que el desarrollo de una práctica profesional esté intrínsicamente marcado por matices y características individuales de cada profesional que la ejecuta, comprendemos la importancia y la necesidad de la construcción de prácticas representativas de características que demarcan la singularidad de nuestra categoría profesional. No defendemos aquí el desarroIlo de una práctica universal pautada en una uniformidad de acciones y percepciones teóricas prácticas. Creemos en la necesidad del desarrollo de acciones reproducidas y fundamentadas en principios, valores y creencias básicas de Terapia Ocupacional en el intento de que, independiente del campo de acción, de la po- blación atendida y de los fundamentos teóricos, podamos reconocernos unos con los otros.

Concluimos que, necesitamos reconocer entre nosotros mismos, como deseamos ser comprendidos y reconocidos por el equipo, por los pacientes y por la sociedad como un todo. Para ello, consideramos fundamental el desarrollo de una identidad colectiva, que represente cada profesional y la categoría de una manera general.

Creemos que estudios tanto en el campo epistemológico como en el clínico son necesarios para el desarrollo de interlocuciones que partan del entendimiento que la Terapia Ocupacional brasileña, y particularmente, el desarrollo de la Terapia Ocupacional en la región amazónica es singular, teniendo mucho que contribuir para el desarrollo de la profesión en el escenario regional, nacional e internacional.

\section{AgRAdeCIMIENTOS}

A los terapeutas ocupacionales que amablemente nos han permitido entrar en sus universos profesionales y contribuyeron a esta investigación.

\section{Referencias Bibliograficas}

(1) HAGEDORN R. Herramientas para la práctica en Terapia Ocupacional: un abordaje estructurado a los conocimientos e procesos centrales. São Paulo: Roca, 2007; 477.

(2) CLARK F, LAWLOR M. The Making and Mattering of occupational science. En: CREPEAU, E, COHN E, SCHELL B. Willard \& Spackman/ Occupational Therapy. 11ed Philadelphia: Wolters Klover Lippincott Williams \& Wilkins, 2009; 02-14.

(3) CLARK F, PARHAN D, CARLSON M, FRANK G, JACKSON J, PIERCE D et al. Occupational Science: academic Innovation in the service of occupational therapy's future. American Journal of Occupational Therapy. 1991; 45: 300-310. 
(4) LIMA E. Identidad y complejidad: composiciones en el campo de la Terapia Ocupacional. Revista de Terapia Ocupacional de la Universidad de San Paulo.1999; 10: 42-45.

(5) MÂNGIA E. Apuntamientos sobre el campo de la Terapia Ocupacional. Revista de Terapia Ocupacional de la Universidad de San Paulo. 1998; 9: 5-13.

(6) MEDEIROS M. Terapia Ocupacional: un enfoque epistemológico y social. São Carlos: EdUFSCAR, 2003; 173.

(7) TURATO Y. Métodos cualitativos y cuantitativos en el área de la salud: definiciones, diferencias y sus objetos de investigación. Revista de Salud Pública. 2005; 39: 507-514

(8) COltRO A. A fenomenología: un enfoque metodológico para más allá de la modernidad. Cuadernos de investigaciones en administración. 2000; 1: 37-45.

(9) GUARNICA A. Algumas notas sobre pesquisa qualitativa e fenomenologia. Revista Interface: Comunicação, Saúde, Educação. 1997; 1:109-122.

(10) GOMES R. La análisis de los datos en investigación cualitativa. En: MINAYO M. Investigación social: teoría, método y creatividad. Petrópolis: Voces. 2001; 67-80.

(11) ARAUJO L. Hermenéutica gestáltica del abuso sexual para una adolescente. 2007. 117f. Disertación (Master en Psicología Clínica e Social) - Universidad Federal de Pará: PPGP, 2007.

(12) OLIVEIRA I. Teciendo el saber: fenomenología del tratamiento de la dependencia química. 2007. 117f. Dissertação (Mestrado em Psicologia Clínica e Social) - Universidade Federal de Pará: PPGP, 2007.

(13) PIMENTEL A, PEDROSO J. Relaciones entre el masticación, alimentación y desarrollo emocional infantil. Revista Paulista de Odontología. 2004; 2: $17-23$.

(14) MEDEIROS M. Terapia Ocupacional con relación a la producción de conocimiento. Cuadernos de Terapia Ocupacional de la UFSCAR. 2000; 6: 6168.
(15) BENETTON J. Trillas Asociativas: ampliando los recursos en la clínica de la Terapia Ocupacional. 3ed. San Paulo: Salesiano, 2006; 144.

(16) SOARES L. Historia de la Terapia Ocupacional. En: CAVALCANTI A, GALVÃO C. Terapia Ocupacional: fundamento y practica. Río de janeiro: Guanabara \& Koogan, 2007: 03-08.

(17) CANIGLIA M. Terapia Ocupacional: un enfoque disciplinar. Minas Gerais: Ophicina Arte \& prosa, 2005; 180.

(18) AMERICAN OCCUPATIONAL THERAPY ASSOCIATION. Occupation. American Journal of Occupational Therapy, 1995; 49: 1015-1017.

(19) LENTIN P. Occupational Terminology Interative Dialogue. Journal of Occupational Science. 2004; 12: 51-53.

(20) HINOJOSA J, KRAMER P. Fundamental concepts of occupational therapy: occupation, purposeful activity and function. American Journal of Occupational Therapy. 1997; 51: 86-866.

(21) TOYODA C, AKASHI L. Actividad: concepto y utilización por los terapeutas ocupacionales - docentes del Estado de San Paulo. Cuadernos de Terapia Ocupacional UFSCar. 1993; 4: 26-35

(22) AMERICAN OCCUPATIONAL THERAPY ASSOCIATION. Framework Occupational Therapy Practice: Domain and Process. 2 ed. American Journal of Occupational Therapy. 2008; 62: 625-683

(23) IWANA M. Toward culturally relevant epistemologies in occupational therapy. American Journal of Occupational Therapy. 2003; 57: 582-588.

(24) IWANA M, SIMÓ S. Aspectos de significado, cultura e inclusión en Terapia Ocupacional. Revista de Terapia Ocupacional de Galícia. 2008;5. Disponible en: http://www.revistatog.com/num8/pdfs/modelo1.pdf (Consultado en 26 de Julho 2009).

(25) CASTRO E, BRUNELLO M, LIMA E. Actividades Humanas y Terapia Ocupacional. En: DE CARLO M, BARTALOTTI C. Terapia Ocupacional en Brasil: fundamentos y perspectivas. San Paulo: Plexus, 2001; 41-59. 
(26) HASSELKUS B. The world of everyday occupation: real people, real lives. American Journal of Occupational Therapy, 2006; 60: 627-640.

(27) PELOQUIN S. História de la Terapia Ocupacional. En: NEISTADT M, CREPEAU E. Willard\&Spackman/ Terapia Ocupacional. Río de Janeiro: Guanabara \& Koogan, 2002; 10-17

(28) LAW M, STEINWENDER S, LECLAIR L. Occupation, health and well-being. Canadian Journal of Occupational Therapy.1998; 65: 81-91.

(29) CLARK F, JACKSON J, SCOTT $M$, CARLSON M, ATKINS M, UHLES-TANAKA D, RUBAYI S. Databased models of how pressure ulcers develop in daily-living contexts of adults with spinal cord injury. Archives Psychiatric Medicine rehabilitation. 2006; 87: 1516-1525.

(30) JACKSON J, CARLSON M, MANDEL, D, ZEMKE R. Occupation in lifestyle redesign: the well elderly study occupational therapy program. American Journal of Occupational Therapy. 1998; 52: 326336.

(31) RUDMAN D, COOK J, POLATAJKO H. Understanding the potential of occupation: a qualitative exploration of senior's perspectives on activity. American Journal of Occupational Therapy.1997; 51: 640-650.

(32) YERXA E. Authentic Occupational Therapy. American Journal of Occupational Therapy. 1967; 21: 155-173. 